See Article page 192.

\section{Commentary: Transcervical left ventricular assist device outflow anastomosis: A top-down approach to sparing the sternum}

\author{
Walker M. Blanding, $\mathrm{MD},{ }^{\mathrm{a}}$ and Arman Kilic, $\mathrm{MD}^{\mathrm{b}}$
}

Although outcomes of left ventricular assist device (LVAD) therapy have improved with newer devices and better patient management, postoperative complications still limit its broader applicability to the ever-increasing heart failure population. As such, there remains an appropriately persistent push for innovation in this field, not just in terms of technology but also in surgical technique and postoperative care. Operative approaches for LVADs have indeed evolved from traditional median sternotomy to less-invasive techniques such as left thoracotomy with upper partial sternotomy as well as total sternal-sparing approaches such as a left thoracotomy combined with right mini-thoracotomy. ${ }^{1,2}$

In addition to mere cosmetic benefit, recent work suggests that less-invasive techniques that preserve the pericardium overlying the right ventricle may lessen the risk of severe right ventricular failure, which is a major predictor of adverse outcomes and mortality in LVAD patients. ${ }^{3}$ In this issue of the Journal, Sutherland and colleagues ${ }^{4}$ present a novel technique detailing a transcervical approach to outflow graft anastomosis to the ascending aorta using the CoreVista system, a single-use submanubrial videoassisted retractor. The paper offers proof of concept for the technique in a cadaveric model.

This approach to the ascending aorta for cardiac surgery has been previously described in surgical aortic valve replacement but has yet to be widely adopted in clinical

\footnotetext{
From the ${ }^{\mathrm{a} D i v i s i o n}$ of Cardiothoracic Surgery, Medical University of South Carolina, Charleston, SC; and ${ }^{b}$ Division of Cardiac Surgery, The University of Pittsburgh Medical Center, Pittsburgh, Pa.

Disclosures: Dr Kilic reported Medical Advisory Board (Medtronic, Inc). Dr Blanding reported no conflicts of interest.

The Journal policy requires editors and reviewers to disclose conflicts of interest and to decline handling or reviewing manuscripts for which they may have a conflict of interest. The editors and reviewers of this article have no conflicts of interest.

Received for publication Feb 15, 2021; revisions received Feb 15, 2021; accepted for publication Feb 22, 2021; available ahead of print March 2, 2021.

Address for reprints: Arman Kilic, MD, University of Pittsburgh Medical Center, 200 Lothrop St, Suite C-700, Pittsburgh, PA 15213 (E-mail: kilica2@upmc.edu).

JTCVS Techniques 2021;7:195-6

2666-2507

Copyright (C) 2021 The Authors. Published by Elsevier Inc. on behalf of The American Association for Thoracic Surgery. This is an open access article under the CC BY-NCND license (http://creativecommons.org/licenses/by-nc-nd/4.0/).

https://doi.org/10.1016/j.xjtc.2021.02.035
}

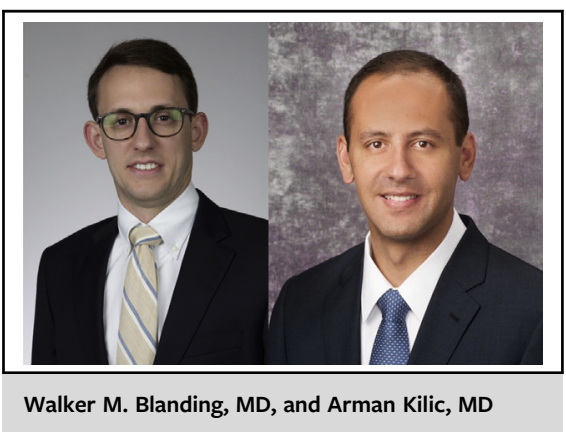

CENTRAL MESSAGE

A novel transcervical approach

to left ventricular outflow graft

anastomosis passes proof-of-

concept testing in a cadaveric

model.

practice. ${ }^{5}$ In addition to more general concerns regarding this approach to the ascending aorta, such as control of bleeding, visualization in patients with short and obese necks, those with ascending aortas that are off-midline, and ergonomics for the surgeon, there are concerns specific to LVAD implantation. For example, maintenance of a sterile field and accommodation of monitoring lines in the cervical area as well as orienting and determining length of the outflow graft to prevent kinking or twisting are relevant obstacles. Despite these concerns, a standard Cooley sidebiting clamp and minimally invasive needle drivers appear to work well for the outflow anastomosis, at least in this cadaveric model. Use of these commonly held instruments also negates the need for ascertainment of further specialized equipment to apply this technique short of the retractor.

While the field is far from knowing the utility of this technique in comparison with other less-invasive techniques already in clinical practice, Sutherland and colleagues' work offers proof of concept for an approach that offers the benefits of sparing the sternum and preserving the pericardium overlying the right ventricle. Additional theoretical benefits of this approach may be avoidance of thoracotomy and its associated postoperative pain, reduced lung volumes, and risk of reintubation, pneumonia, and other respiratory complications.

\section{References}

1. Schmitto JD, Krabatsch T, Damme L, Netuka I. Less invasive HeartMate 3 left ventricular assist device implantation. J Thorac Dis. 2018;10:S1692-5.

2. McGee E, Danter M, Strueber M, Mahr C, Mokadam NA, Wieselthaler G, et al Evaluation of a lateral thoracotomy implant approach for a centrifugal-flow left 
ventricular assist device: the LATERAL clinical trial. J Heart Lung Transplant. 2019;38:344-51.

3. Saeed D, Muslem R, Rasheed M, Caliskan K, Kalampokas N, Sipahi F, et al. Less invasive surgical implant strategy and right heart failure after LVAD implantation. J Heart Lung Transplant. January 12, 2021 [Epub ahead of print].
4. Sutherland F, Gradinariu1 G. Transcervical approach for left ventricular assist device outflow graft: a proof-of-concept implantation study. J Thorac Cardiovasc Surg Tech. 2021;7:192-4.

5. Dapunt OE, Luha O, Ebner A, Sonecki P, Spadaccio C, Sutherland FWH. First-inman transcervical surgical aortic valve replacement using the CoreVista system. Innovations (Phila). 2016;11:84-93. 